\title{
Migraine-Like Positive Visual Phenomena Related to Focal Cortical Lesions with Undetectable Visual Field Defects
}

\author{
Amir Vosoughi ${ }^{a}$ Andrew Micieli ${ }^{b}$ Jonathan A. Micielib, c, d \\ a Max Rady College of Medicine, University of Manitoba, Winnipeg, MB, Canada; ${ }^{b}$ Division \\ of Neurology, Department of Medicine, University of Toronto, Toronto, ON, Canada; \\ 'Department of Ophthalmology and Vision Sciences, University of Toronto, Toronto, ON, \\ Canada; ${ }^{\mathrm{d} K e n s i n g t o n}$ Vision and Research Centre, Toronto, ON, Canada
}

\section{Keywords}

Migraine $\cdot$ Meningioma $\cdot$ Visual aura $\cdot$ Cavernoma $\cdot$ Demyelination

\begin{abstract}
Migraines are commonly associated with a visual aura that has a characteristic clinical presentation. Cortical lesions within or in close proximity to the retrochiasmal visual pathways may also present in a manner that mimics migrainous visual phenomena and, in some cases, may be too small to manifest with a visual field defect on formal testing. We present 4 patients (3 females and 1 male) with an average age of 48.5 (range 28-67) years who had migraine-like visual disturbances related to a right temporal meningioma, occipital cavernoma, occipital lobe infarction, and demyelination in the optic radiations, which was the presenting sign of multiple sclerosis. No patient underwent neurosurgical intervention, and 1 patient (occipital lobe infarct) had complete resolution of the symptom after initial presentation. All patients had normal visual fields at follow-up and no thinning evident on optical coherence tomography. Our cases emphasize the importance of a history in assessing patients with transient positive visual phenomena and identify pathology that may present without visual field defects. Clinical features that should raise a doubt about a diagnosis of migraine visual aura include the absence of headache, brief visual disturbance lasting $<5$ min or those lasting $>60$ min, and age $>40$, especially with no past medical history of migraine.
\end{abstract}

\section{Karger ${ }^{\prime \prime}=$}




\section{Introduction}

Migraines are commonly associated with a visual aura that has a characteristic clinical presentation. Cortical lesions within or in close proximity to the retrochiasmal visual pathways may also present in a manner that mimics migrainous visual phenomena. The majority of patients with positive visual disturbances related to cortical lesions have visual field defects that can be detected by confrontation or formal automated perimetry [1]. The patient may be unaware of these defects in their peripheral vision. In one of the largest studies by Shams and Plant [1], 9 patients were presented with a review of 31 additional cases from the literature. Of the 9 cases in the primary series, 7 had detectable visual field defects and those without a defect were related to a small cavernoma in the occipital lobe and an oligodendroglioma in the left parietooccipital region. The goal of this study was to present 4 patients with new positive visual phenomena and normal Humphrey 24-2 SITA-Fast visual fields to expand the differential diagnosis for cortical lesions that may manifest without detectable visual field defects on formal testing.

\section{Case Reports}

\section{Case 1}

A 56-year-old woman was referred for positive visual phenomena. She was otherwise healthy and did not have a history of migraines or other primary headache disorder. She also had no family history of migraines. One year prior to presentation, she developed new bright, white-colored "zig-zags" in her visual field that always occurred on the left side of her visual field. This appeared similar when she occluded either eye on several occasions. These lasted 2-5 min and occurred once every few weeks for several months. This then decreased in frequency to every couple of months, but consistently of the same semiology on the left side of the visual field. Two weeks prior to presentation, she developed a shimmering light in her left visual field that interfered with her ability to work on the computer. This spread toward the center of her vision and precluded her from continuing her work. After about $20 \mathrm{~min}$, the visual changes resolved and she developed a mild holocephalic headache that lasted the remainder of the day. There was no associated nausea, photophobia, or phonophobia, and it was not worsened by activity. Due to her new visual disturbance, she was referred for an ophthalmology consultation.

Examination revealed a visual acuity of $20 / 20$ in both eyes. Humphrey 24-2 SITA-Fast visual fields were performed and these were normal in each eye. Pupils were equal and reactive to light, and there was no relative afferent pupillary defect. Slit-lamp examination revealed normal appearing anterior chambers, and dilated examination revealed normal appearing optic nerves and retinas. Optical coherence tomography (OCT) of the retinal nerve fiber layer (RNFL) and ganglion cell-inner plexiform layer (GCIPL) were normal. Due to concern for an underlying cortical lesion resulting in left-sided positive visual phenomena, a magnetic resonance imaging (MRI) brain was performed. This revealed a solitary dural-based mass in the right inferior temporal horn measuring approximately $2.5 \times 1.8 \times 2.3 \mathrm{~cm}$, consistent with a meningioma (Fig. 1). The patient had additional workup including a computed tomography (CT) of her chest/abdomen/pelvis, and no concerning lesions for malignancy were found. A neurosurgery consultation was arranged, but the patient opted for observation. Ather 6-month follow-up, she continued to have normal visual acuity and normal visual fields. Her positive visual disturbances reduced in frequency and occurred 2 times over the next 6 months.

Case 2

A 43-year-old man was referred for positive visual phenomena. His medical history was significant for cholecystectomy and he did not take any regular medications. He reported

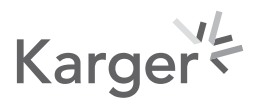



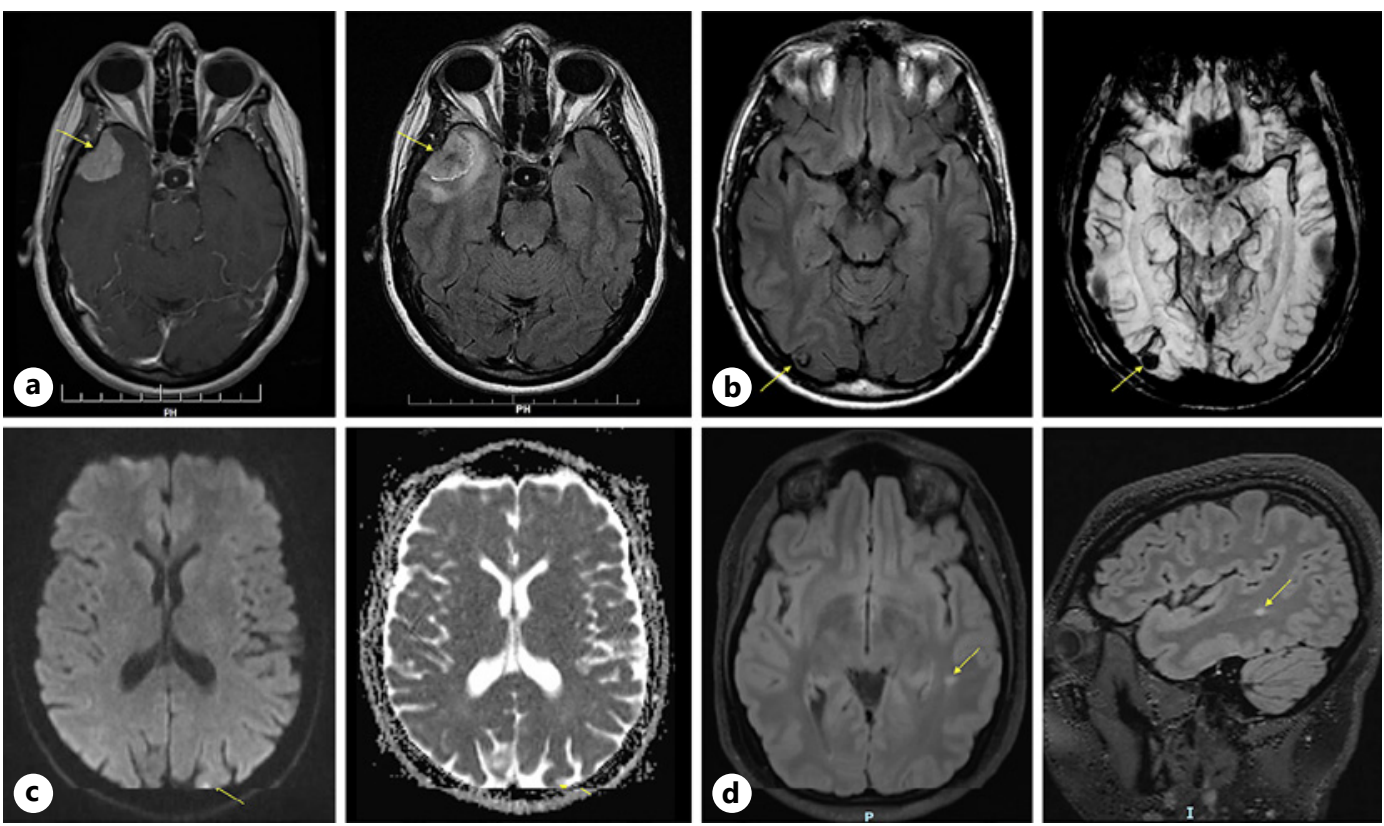

Fig. 1. a Axial MRI T1-post-contrast (left) and FLAIR (right) demonstrating a right temporal lobe meningioma with mild surrounding edema. $\mathbf{b}$ Axial MRI demonstrating a focal subcentimeter T2/FLAIR hyperintense lesion with peripheral hypointensity rim most consistent with a cavernoma (left), which was confirmed on susceptibility-weighted imaging (right). In addition, there was suspicion of an adjacent developmental venous anomaly with small channels and a prominent draining vein. c MRI showing a left superior peripheral occipital lobe $3 \mathrm{~mm}$ focus of diffusion restriction (left) and corresponding hypointensity on ADC (right). d Axial (left) and sagittal (right) MRI FLAIR showing a white matter lesion in the left temporal lobe secondary to multiple sclerosis. MRI, magnetic resonance imaging.

infrequent headaches, occurring 1 or 2 times per year associated with stress or drinking alcohol. On the day of presentation, he developed new flashes of light on the left side of his visual field while working at the computer. This appeared similar when he occluded either eye and lasted approximately $15 \mathrm{~min}$ and was associated with photophobia. His vision returned to normal 5 min later. This episode was not associated with the development of a headache, nausea, or other neurological symptoms. He presented to the emergency department and had a CT scan of the head, which was reported as normal. He was referred to ophthalmology and had a visual acuity of 20/20 in both eyes, and Humphrey visual field testing (24-2 SITA-Fast) was normal without signs of a homonymous hemianopia. Anterior segment and dilated fundus examination was normal. OCT RNFL and GCIPL were normal. Given that he had no history of migraine headache and these new positive visual disturbances were new for him, an MRI of the brain was performed, and this demonstrated a right-sided focal subcentimeter T2/FLAIR hyperintense lesion with peripheral hypointensity rim most consistent with a cavernoma, which was confirmed on susceptibility-weighted imaging. In addition, there was suspicion of an adjacent developmental venous anomaly with small channels and a prominent draining vein. No intervention was recommended, and the patient had one additional episode similar to the previous one associated with left-sided "zig-zag" lights on the left side of his visual field that lasted 15 min two weeks after his initial episode. His visual function remained normal at the 6-month follow-up.

Case 3

A 67-year-old woman was referred to neuro-ophthalmology for positive visual phenomena after a cardiac angiogram. She had a medical history of hypertension, and her 
medications included aspirin $81 \mathrm{mg}$, amlodipine, and candesartan. She developed new exertional chest pain and was found to have an abnormal cardiac stress test. This prompted a coronary catheter angiogram, and $1 \mathrm{~h}$ after completion of the procedure, she noticed flashing lights on the right side of her visual field that lasted several hours. She denied headache, nausea, photophobia, or other focal neurological symptoms. Urgent ophthalmology consultation revealed a visual acuity of 20/20 in both eyes, and Humphrey 24-2 SITA-Fast visual fields were performed and were normal in both eyes without any signs of homonymous changes. The anterior segment was normal, and dilated fundus examination revealed normal-appearing optic nerves and retinas. OCT, RNFL, and GCIPL were normal. Given the length of her positive visual disturbance and the temporal relationship to her catheter angiogram, an MRI and MRA of the brain were performed and showed a left superior peripheral occipital lobe $3 \mathrm{~mm}$ focus of diffusion restriction, and no significant extra-/intracranial stenosis. The patient underwent additional testing including transthoracic echocardiogram and 48-h Holter monitor that were normal. The etiology of the stroke was presumed cardioembolic secondary to the cardiac angiogram. She had no additional positive visual disturbances, and she continued to have normal visual fields at the 6-month neuro-ophthalmology follow-up.

\section{Case 4}

A 28-year-old woman was referred to neuro-ophthalmology for transient blurred vision on the right side of her visual field. She was healthy and did not have any known medical conditions. Two weeks prior to presentation, while watching television, she noticed that the right side of her visual field gradually became blurry over $5 \mathrm{~min}$. This persisted for $45 \mathrm{~min}$ and was associated with a "pulsing" light in the superior part of her right visual field. She occluded either eye and the right side of her visual field appeared similar in either eye. There was no associated headache afterward. She had a similar episode 2 weeks later that also lasted $45 \mathrm{~min}$ but was not associated with the "pulsing" light. There was no other history of previous transient neurological symptoms. She presented to the emergency room and had a CT scan of the head that was reported as normal, and she was referred to ophthalmology.

On exam, her visual acuity was 20/20 in both eyes, and Humphrey 24-2 SITA-Fast visual fields were normal in both eyes. Dilated fundus examination and neurological examination were also normal. OCT, RNFL, and GCIPL were normal. Given that she had 2 episodes of transient visual changes always on the right side of her visual field, an MRI of the brain with contrast was arranged to assess for a left retrochiasmal visual pathway lesion. This demonstrated multiple T2/FLAIR hyperintense lesions in the deep and periventricular white matter most consistent with multifocal demyelination. There were no infratentorial or contrastenhancing lesions. There was a lesion within the left optic radiations that was thought to be the etiology of the right-sided visual disturbances. The presentation was most consistent with demyelination, and a 4-month follow-up MRI showed a new right periventricular lesion, thereby fulfilling McDonald criteria for multiple sclerosis. At the 6-month neuro-ophthalmology follow-up, she had no additional visual episodes and her visual function remained normal.

\section{Discussion}

Our case series of 4 patients demonstrates that focal cerebral lesions may present with isolated positive visual disturbances that may resemble the visual aura associated with migraine. Although previous studies have demonstrated that most of these lesions have a detectable visual field deficit, formal Humphrey visual fields may be normal in these patients.

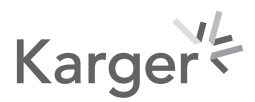


A careful history is therefore required when deciding to perform neuroimaging when the visual field is normal as obtaining neuroimaging in all patients with positive visual disturbances is not practical. Several features in our patients raised concern for an underlying structural lesion. Visual aura recurring in the same hemifield (cases 1 and 4), absence of a migraine history (all cases), and long duration of the visual aura (case 3) were instrumental in the decision to perform neuroimaging. Case 1 had very brief episodic changes consistent with visual seizures that preceded the longer migrainous disturbance that lasted $20 \mathrm{~min}$. Case 3 also had a cardiac angiogram in close temporal relationship to her visual disturbance, which raised concern for an ischemic infarct. As previously described [1], clinical features that should raise a doubt about a diagnosis of migraine visual aura include the absence of headache, brief visual disturbance lasting $<5 \mathrm{~min}$ or those lasting $>60 \mathrm{~min}$, and age $>40$ years, especially with no past medical history of migraine. A stereotyped aura always in the same hemifield or a persistence of a scotoma or visual field defect should prompt neuroimaging. In those with a history of a long-standing visual aura, a change in frequency or characteristic of the aura also warrants further investigation with MRI.

Structural lesions are hypothesized to cause migraine-like auras by various processes, resulting in neuronal hyperexcitability [1]. Such states lower the threshold for spontaneous neuronal depolarization and thereby leave the brain susceptible to cortical spreading depression- a slow wave of neuronal and glial depolarization followed by a state of inhibition. Previous cases of meningiomas mimicking migraine with visual aura have been reported and typically resolve postoperatively, but formal visual fields were not reported or performed in most cases [1]. The presence of a visual field defect is provided only in 2 of the cases, a bitemporal hemianopia that resolved postoperatively [2] and a right inferior homonymous quadrantanopia [3] which remained postoperatively. In the review by Shams and Plant [1], 16 out of 31 cases were identified as AVMs. Additional cases of AVMs and positive visual disturbances have been reported in the literature with few of these cases presenting with a normal visual field when it was performed, likely due to the small size of the lesion or presence just outside of the visual pathways [1]. The migraine and aura improved in 3 of the 4 cases following surgery [4]. New migraine headache and worsening of migraine headaches have been reported to be the initial manifestation of MS or an MS relapse. We were unable to retrieve a previous case report of migrainous visual disturbances or migraine with aura as the presenting sign of MS as seen in our patient. Moreover, cerebral ischemia is likely to lower the threshold for developing migraine-like aura, and previous studies have demonstrated that stroke may initially present with symptoms resembling migraine aura [5]. Visual fields were not routinely performed in these studies [5].

The cases provided in this report provide additional evidence that normal formal automated perimetry with 24-2 Humphrey testing is not sufficient to rule out a focal cortical lesion. In each case, the history was highly characteristic as the visual disturbances were contralateral to the lesion in all cases and had atypical features for classical migraine visual auras. However, a cause and effect relationship could not be firmly established with the exception of case 3 who had no additional visual changes after her acute stroke. The other patients did not undergo surgical excision of the lesions since the risk/benefit ratio was considered to be too large.

In conclusion, positive visual disturbances of central origin are a common presenting complaint to ophthalmologists, of which the vast majority can be attributed to migraine. This case series highlights various pathologies including meningioma, cavernoma, ischemia, and demyelination that may not produce detectable visual field defects. This emphasizes the importance of a careful history and understanding that a normal visual field may not rule out an underlying cortical defect. MRI of the brain is the recommended next step when a cortical lesion is suspected.

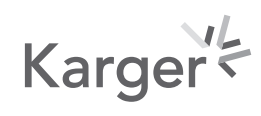




\section{Acknowledgments}

This manuscript does not include any nonauthor contributors to acknowledge.

\section{Statement of Ethics}

Research Ethics Board approval was not required for this study from our institution. Written informed consent was obtained from the patients for publication of the details of their medical case and any accompanying images.

\section{Conflict of Interest Statement}

The authors of this manuscript do not have any conflict of interest to declare.

\section{Funding Sources}

This study did not receive any funding.

\section{Author Contributions}

Conception and design: A.R.V., A.M., and J.A.M.; acquisition of data: A.M. and J.A.M.; draft of the manuscript: A.R.V.; critical revisions: A.M. and J.A.M.; and final approval: A.R.V., A.M., and J.A.M.

\section{Data Availability Statement}

All data generated during this study are available. Further enquiries can be directed to the corresponding author.

\section{References}

1 Shams PN, Plant GT. Migraine-like visual aura due to focal cerebral lesions: case series and review. Surv Ophthalmol. 2011 Mar-Apr;56(2):135-61.

2 Schlake HP, Grotemeyer KH, Husstedt IW, Schuierer G, Brune GG. "Symptomatic migraine": intracranial lesions mimicking migrainous headache - a report of three cases. Headache. 1991;31(10):661-5.

3 Vetvik KR, Dahl M, Russell MB. Symptomatic sporadic hemiplegic migraine. Cephalalgia. 2005;25(11):1093-5.

4 Kupersmith MJ, Berenstein A, Nelson PK, ApSimon HT, Setton A. Visual symptoms with dural arteriovenous malformations draining into occipital veins. Neurology. 1999;52(1):156-62.

5 Olesen J, Friberg L, Olsen TS, Andersen AR, Lassen NA, Hansen PE, et al. Ischaemia-induced (symptomatic) migraine attacks may be more frequent than migraine-induced ischaemic insults. Brain. 1993;116(Pt 1):187202.

\section{Karger'א}

